Pediatr. Res. 16: 1048 (1982)

\title{
Author Index to Abstracts
}

\author{
Al-Zubaadi IM 20 \\ Ameghio $\mathrm{F} 42$ \\ Ansaldi N 14 \\ Aramayo L 50 \\ Arhan P 12 \\ Arnaud-Battandier F 23 \\ Auricchio S 13, 14, 42 \\ Ballabriga A 10 \\ Beguinot L 13 \\ Benderly A 43 \\ Berant M 4 \\ Bernardin JE 13 \\ Biemond I 39, 40 \\ Bijleveld CMA 6, 38 \\ Blackshaw AJ 47 \\ Boccon-Gibod L 55, 57 \\ Bogoniowska Z 33 \\ Booth IW 21 \\ Born M 58 \\ Borulf S 26 \\ Bossi A 14 \\ Bouquet J 40 \\ Boyle S 47 \\ Bremer HJ 20 \\ Bresson JL 19 \\ Brick R 4 \\ Brink EAA 49 \\ Brinkman B 49 \\ Brown GA 50 \\ Brueton MJ 24 \\ Brügmann G 37 \\ Buffolano W 42 \\ Buis JJ 49 \\ Cadranel S 27 \\ Canarelli JP 11, 12 \\ Candy DCA 29 \\ Capano G 28, 53 \\ Caprioli A 53 \\ Carocho MC 11 \\ Carrasco S 39, 41 \\ Castano C 34 \\ Castelpietra L 36 \\ César JP 5 \\ Chapoy P 56 \\ Chatelet F 57 \\ Chong SKF 47 \\ Ciccimarra $F 42$ \\ Clayton PT 35 \\ Codoceo R 1, 34 \\ Cortinovis L 14 \\ Coutance F 46 \\ Cucchiara S 53 \\ Dall'Olio D 14 \\ Dawson AM 47 \\ deJose MI 1 \\ deMol P 27 \\ dePrez C 27 \\ deRitis G 4, 13, 53
}

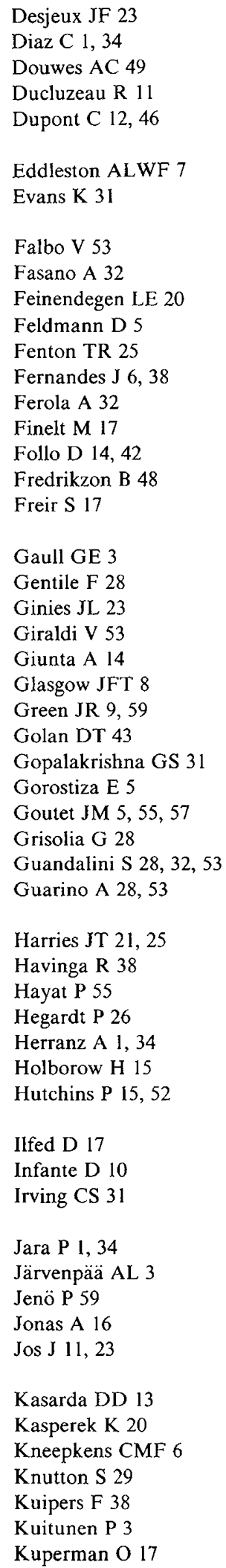

Räihä NCR 3

Rantala I 30

Rassin P 3

Rey J 23

Ricour C 11, 23

Rodesch P 27

Rosenthal E 43

Rossipal E 44

Roux F 56

Rubino A 28, 32, 53

Savilahti E 18

Schmidt E 58

Schmitz J 11, 23

Schorel E 40

Schreuder GMTh 39, 40, 41

Siimes $S 18$

Sinaasappel M 40

Socha J 33

Solberg Scott M 7

Sonsino E 12

Spitz L 25

Stenling R 48

Sterchi EE 9

Szeligowski M 33

Szymanski W 33

Tamaro G 36

Tanner MS 51

Teufel M 37

Tiribelli C 36

Tormo R 10

Torre G 36

Tosi R 42

Trevisan M 36

Triadou N 11, 23

Unsworth DJ 15

Navarro J 5, 12, 55, 57

Nichols BL 31

Niessen KH 37

Nixon HH 25

Nouri K 7

Oren M 16

Passwell J 16

Pawlowska J 33

Pena AS 39, 40, 41

Perini R 36

Perkkiö M 18

Phillips AD 15, 45, 52

Ploussard JP 57

Polanco I 39, 4 I

Folonovski C 5, 51

Pompidou A 46

Portmann B 2

Prampolini L 14

Prieto G 39, 41

Pscharopoulos HT 2
Van Gelderen HH 40

Van Oeveren JP 49

Van Rood JJ 39, 40, 41

Vazquez C 1, 34, 39, 41

Vergani D 7

Vesikari T 30

Virelizier JL 55

Visakorpi JK 30

Vismara D 42

Vogel A 49

Vonk RJ 6, 38

Vukavic T 54

Walker AW 19

Walker-Smith JA 15, 47

Walker-Smith J 52

Warren IF 51

Weil S 17

Williams CB 47

Wolff $\mathrm{OH} 22$ 